\title{
Effect of solvent lamination on roll-to-roll hot-embossed PMMA microchannels evaluated by optical coherence tomography
}

\author{
Janne Lauri $^{1 *}$, Christina Liedert ${ }^{2}$ Annukka Kokkonen ${ }^{2}$ and Tapio Fabritius ${ }^{1}$
}

1. Optoelectronics and Measurement Techniques Unit, Faculty of Information Technology and Electrical Engineering, University of Oulu, P.O. Box 4500, FI-90014, Oulu, Finland;

2. VTT Technical Research Centre of Finland Ltd, Kaitoväylä 1, P.O. Box 1100, 90571 Oulu, Finland

*Correspondence: janne.lauri@oulu.fi

\begin{abstract}
Manufacturing of microfluidic based diagnostic devices requires small tolerances and uniform quality to guarantee reliable and repeatable test results. This work describes characterization of morphological changes that occur to a hot embossed PMMA microfluidic channel after solvent lamination with a PMMA lid. A noncontact cross-sectional analysis of the lidded microfluidic device was performed by optical coherence tomography (OCT). The solvent induced morphology change led to a porous structure in bottom corners of hot-embossed channels, which allowed a fluid to absorb in the material. The measurements of solvent diffusion showed faster diffusion rate at the corners of the channel, in which the accumulated stress during the embossing process was the highest. The stress profile was verified by simulation of von Mises stresses during a molding phase of a hot embossing process. The porous structure with increased fluid diffusion has an unwanted effect on bioassay result, e.g. when detection molecules leak into the substrate thus leading to unspecific signal on chip. OCT was found to be a valuable, non-destructive imaging method to monitor solvent diffusion process and lamination process quality.
\end{abstract}

Keywords: diffusion rate, solvent bonding, porous PMMA, microfluidics

\section{Introduction}

Microfluidics enables precise control of volumes and flow of fluids [1]. Technology allows small quantities of biological samples and reagents to be moved, mixed and detected with high accuracy and speed. Thus, the technology holds great promise for point-of-care diagnostics as complex laboratory procedures and instruments can be minituarized into a single microchip.

Hot embossing technology is a low cost, flexible fabrication method, which allows fast and accurate replication of high aspect ratio micro and nanostructures on a large area [2]. In the process, a polymer substrate such as PMMA (polymethyl methacrylate) and COC (cyclic olefin copolymers), is heated over the glass transition point and pressed against a master stamp imprinting a replica. The throughput of the method can be further increased by roll-to-roll (R2R) hot embossing process where polymer web is continuously fed through a pressurized area between embossing cylinder and a blank counter cylinder $[3,4]$.

The hot embossed microfluidic channels have to be lidded prior use. There exists various bonding methods to attach polymer layers together: thermal diffusion bonding, pressure-induced sealing, adhesive bonding, thermal lamination, laser welding, surface treatment and conventional solvent bonding [5-8]. The solvent bonding/lamination is one of the potential methods to be used in roll-to-roll process and mass production. In the solvent lamination process of glassy polymers, such as PMMA and COC, typically non-Fickian diffusion (case-II) occurs when temperature is below glass transition point [5,9-11]. In this diffusion process solvent penetrates into the substrate with a sharp boundary of swollen layer and unpermeated glassy core. The mobile polymers chains in the swollen layer diffuse and intertwine with the chains between the surfaces resulting a very strong bond [6]. The rate that the swollen layer forms is affected for instance by medium composition, immersion time and temperature [5]. During a molding phase of the embossing process the substrate 
experiences high stress concentrations points and strains at the stamp corners [12,13] as well as material flow [14], which can change the substrate composition and further affect the swollen layer formation rate. Depending on the solvent-polymer interaction, dissolution process of the polymer can be uncomplete and result a formation of a plasticized matrix [5]. If the matrix is porous it causes problems in a microfluidic operation and effects on the reliability of the analysis. For instance, a porous structure may lead to false bioassay results, e.g. when detection molecules leak into the substrate leading to unspesific signal amplification on chip.

The effects of solvents on polymers are well studied [9,15-17]. Various experimental techniques, such as differential refractometry, laser interferometry, optical microscopy, ellipsometry and critical angle illumination microscopy $[17,18]$, have been applied to study penetration and dissolution kinetics. These techniques are mostly applicable to study only thin films with the thickness of one micron or require a special sample cell. Depth resolved characterization of dissolution process, for instance during solvent lamination process, is unfeasible. A non-destructive, real-time imaging modality, optical coherence tomography (OCT), has enabled micrometer scale visualization of a scattering media and flow. The utilization and the potential of OCT has been well recognized in the field of rheology and microfluidics [19-26], ophthalmology [27] as well as non-destructive testing $[28,29]$. OCT detects light that is backscattered from the material inhomogeneity i.e. refractive index change. Thus, it can be used to investigate crystallization processes in polymers[30] and for instance dissolution process during solvent lamination and crazing in polymer substrates.

In this study, we measure changes in morphology of hot embossed microfluidics on PMMA substrate after solvent lamination. The swollen layer formation rate at walls of the microchannel is visualized, for the first time to our knowledge, with OCT. We experimentally validate the existence of the local porous structure, which will an impact on a readout of a fluorescent signal if excess of fluorescent labels and reagents cannot be washed out. Simulation of hot-embossing process is performed to the estimated relation of the porous structures to high stress and strain locations. We demonstrate that OCT is excellent tool for quality assurance of complete microfluidic channels and to monitor dissolution process in polymer substrates.

\section{Materials and measurement methods}

\section{Hot embossing of microfluidic channels on PMMA foil}

The microfluidic channels were fabricated by roll-to-roll hot embossing (Fig. 1a) on thermoplastic polymethyl methacrylate (PMMA) foil (Plexiglass 99524, Evonik Röhm, Germany) with a web width of $200 \mathrm{~mm}$ and thickness of $375 \mu \mathrm{m}$. The microfluidic channels ( $400 \mu \mathrm{m}$ in width) were drawn with a commercial software (Rhino3D from McNeel, Spain) from which large area steel shim was fabricated by wet etching as described in ref. [4]. The steel shim was laser welded into a closed cylinder and installed to hot embossing unit of the roll-to-roll pilot line (Fig. 1b). The embossing parameters were $105^{\circ} \mathrm{C}$ for embossing cylinder and $85{ }^{\circ} \mathrm{C}$ for counter cylinder surface. The nip pressure and web speed were set as described in results.

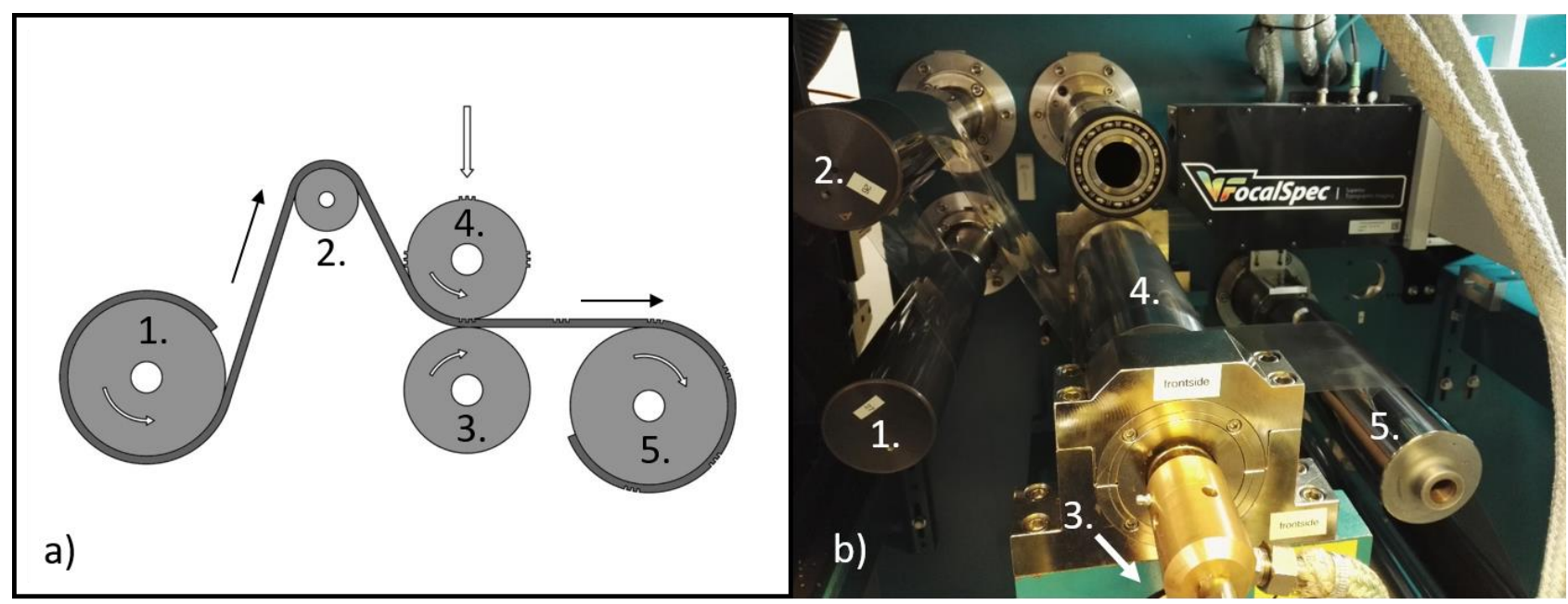

Figure 1 Roll-to-roll hot embossing process. a) The thermoplastic polymer web is continuously fed with 
constant speed and pressure between a microstructured embossing cylinder and counter pressure cylinder (arrow). 1) Unwinder (where the blank material comes from), 2) web path control, 3) counter pressure cylinder, 4) hot embossing cylinder, 5) rewinder (where the hot embossed material is rolled). The cylinders are heated individually by oil circulation pumps. b) Image of the embossing unit at printing pilot line.

\section{Lidding of microfluidic channels with solvent bonding}

The embossed channels were cut out from the web and laminated individually with the help of a contact coater (K Control coater 202, R K Print-Coat Instruments Ltd, UK). In short, the inlet and outlet vias (diameter 2mm) were drilled on the PMMA cover layer (thickness $175 \mu \mathrm{m}$, Plexiglass 99524, Evonik Röhm, Germany) and aligned manually with cut out PMMA channels. Two layers were first bonded from the upper parts with a strip of office tape and set to the control coater so that the cover lid was bend upwards under a metal rod leaving an open gap between layers. $400 \mu 1$ solvent mixture (25\% EtOAc: $75 \%$ IPA) was pipeted between layers after which cover lid was allowed to unroll on top of the channel layer with electronically driven metal rod (speed $14 \mathrm{~cm} \mathrm{~s}^{-1}$ ). The excess solvent remaining within the microfluidic channel was removed by water driven suction hose. The bonded devices were allowed to dry overnight.

\section{Optical coherence tomography}

Structural characterization was done with the OCT device (Hyperion, Thorlabs Inc.). The axial (depth) and the lateral resolution of the system are 5.8 (in air) $\mu \mathrm{m}$ and $8 \mu \mathrm{m}$ respectively. By lateral scanning of a probing beam structural intensity images of cross-sections of microfluidic channels are acquired. In axial direction OCT measures optical path length differences. To obtain actual distances inside a sample images all of the images are scaled with the refractive index (n) of water (1.33). The probing beam was set in a small angle in relation to the microfluidic chip surface to reduce the strong backscattering from chip surface and improved the sensitivity of the measurements inside the chip.

\section{Results and discussion}

\section{Hot-embossed microchannels}

Before the solvent lamination, the cross-section of the microchannel was characterized (Fig. 2a). By evaluation of the scattering intensity and its uniformity inside the PMMA substrate, the morphology appeared homogeneous. After the solvent bonding with the contact coater scattering areas appear below the corners of the microchannel (see arrows in Fig. 2b). The areas are well defined and extent several tens of micrometers deep. Because OCT signal originates from the refractive index changes in the material, such a strong signal indicates that there is either heterogeneous material or there are air voids. To investigate possible existence of air voids; the microchannel was filled with water (Fig. 2c). If the scattering sites are porous they will absorb water and result attenuation of OCT signal due to the optical clearing effect that evens a refractive index mismatch between the voids and a surrounding PMMA. Because the backscattered signal intensity in OCT is proportional to the difference in refractive indexes of two materials, the larger difference in refractive indexes results a higher signal intensity. If the refractive indexes are almost matching, backscattered signal becomes too low to be detected by OCT. After 1 min of incubation of water the scattering areas start to vanish (Fig. 2d) and after $5 \mathrm{~min}$ (Fig. 2e) they are almost completely disappeared. Such a result indicates that the scattering sites consist mainly of porous PMMA. The air voids $(n=1)$ are filled with absorbed water $(n=1.33)$ which leads to reduced light scattering intensity and cannot be detected anymore by OCT. Origin of the porous structure is assumed to be a result of the ceased swelling-evaporation processes [31-33]. 



Figure 2 The cross-section of microfluidic channel. a) Unlidded channel, b) empty channel with scattering sites (arrows), c) filled with water $(\mathrm{t}=0 \mathrm{~min}), \mathrm{d})$ filled with water $(1 \mathrm{~min}$ of incubation) and e) filled with water (5 min of incubation). Embossing parameters were $30 \mathrm{bar}, \mathrm{v}=0.5 \mathrm{~m} / \mathrm{min}, \mathrm{T}$ (embossing cylinder) $=$ $105^{\circ} \mathrm{C}, \mathrm{T}$ (counter cylinder $)=85^{\circ} \mathrm{C}$.

\section{Response of PMMA to ethanol acetate}

Previously proved the existence of voids and their location in the bottom corners of the laminated channel structure shows that dissolution rate appears to be nonhomogeneous. This indicates that the solvent diffusion rate is somewhat influenced by the hot embossing. To verify this, the microfluidic channel was filled with solvent (99.6\% EtOAc) and covered with a piece of PMMA film (Fig. 3). The reason to use pure ethanol acetate as a solvent was due to the fact that the original solution (25\% EtOAc: $75 \%$ IPA) appeared to require additional pressure to initiate a dissolution process. After adding the solvent, the piece of PMMA film was placed over the microchannel and cross-sectional images were recorded at $5 \mathrm{~s}$ intervals up to $4 \mathrm{~min}$.

Figure 3 at $\mathrm{t}=0 \mathrm{~s}$ shows the microchannel at the moment when the channel was filled with the solvent. At this point there is no indication of a beginning of a dissolution process or swelling layer. At the $t=25 \mathrm{~s}$ dissolution process has clearly started from the bottom corners of the microchannel. In addition, the solvent begins to scatter light, which indicates that parts of the polymer molecules are detached from the solid surface and dispersed into the solvent. After $50 \mathrm{~s}$ of incubation the sharp front of the dissolution process evolves much faster at the bottom corners of the microchannel. After $100 \mathrm{~s}$ the leading edges of the swollen layer at the bottom corners get deeper and wider. Finally, also so the middle bottom part is almost completely dissolved after $200 \mathrm{~s}$. 

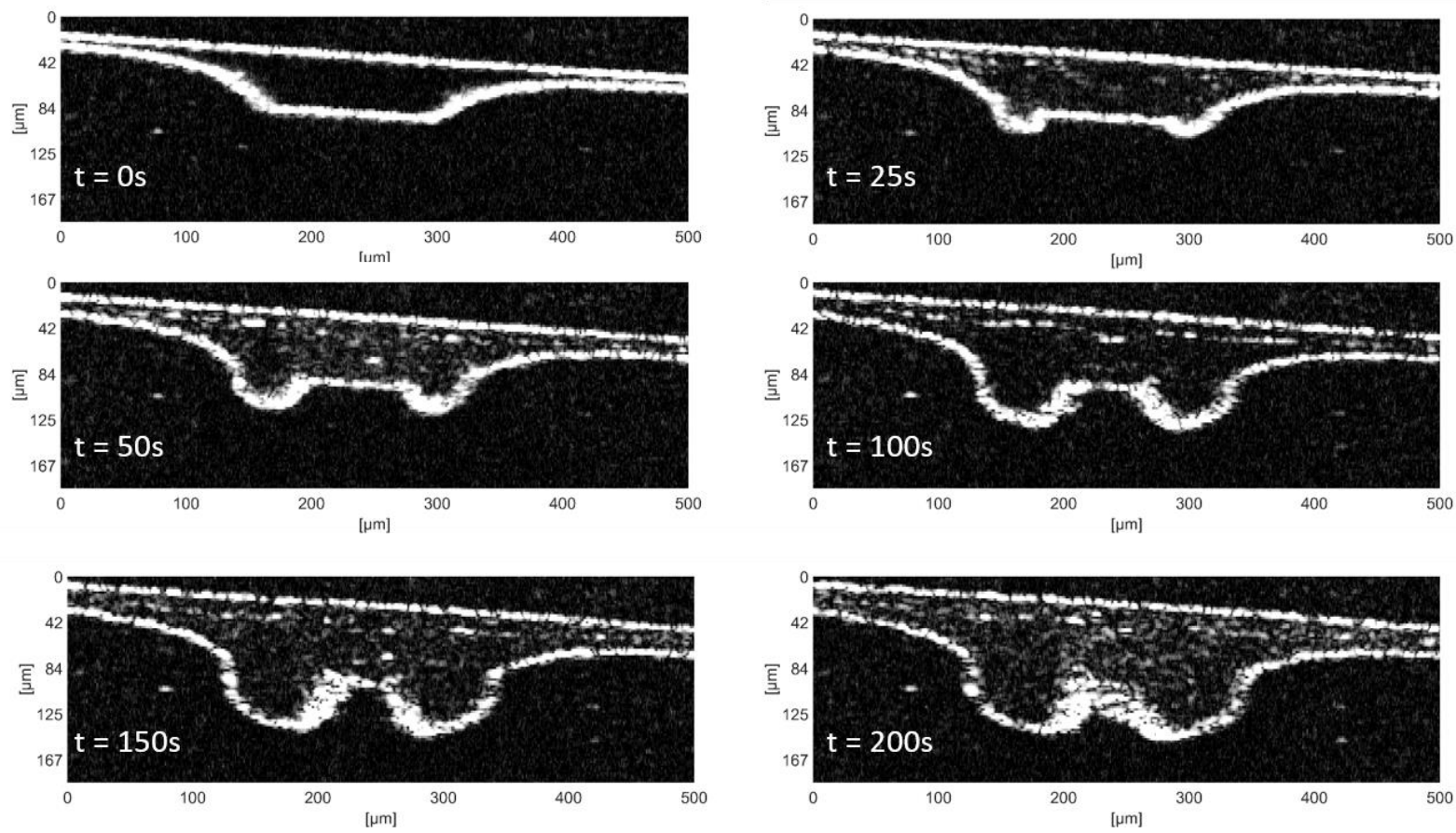

Figure 3 Evolution of the dissolution process in the microfluidic channel filled with EtOAc (99.6\%). At the beginning the solvent is clear and channel is unaffected. After $25 \mathrm{~s}$ of incubation the solvent has dissolved mostly the bottom corners and detached polymer molecules makes solvent more scattering. The faster dissolution at the bottom corners becomes more evident at the times: $50 \mathrm{~s}, 100 \mathrm{~s}$ and $150 \mathrm{~s}$. After $200 \mathrm{~s}$ the middle bottom part is almost completely dissolved

While the lid was placed on top of the microchannel, some air bubbles were trapped into the channel (Fig. 4a). At these locations the channel is nearly empty and most of the solvent located only at the sidewalls. In Figure $4 \mathrm{~b}$, the cross-sectional image is measured at point of on a trapped air bubble. The solvent has diffused into PMMA mainly at the bottom corners and films' interface, however, the middle bottom part of the microchannel is not dissolved. A shape of the microchannel is retained after $200 \mathrm{~s}$. Scattering sites, emerged in the bottom corners, are similar to those ones laminated by the contact coater.
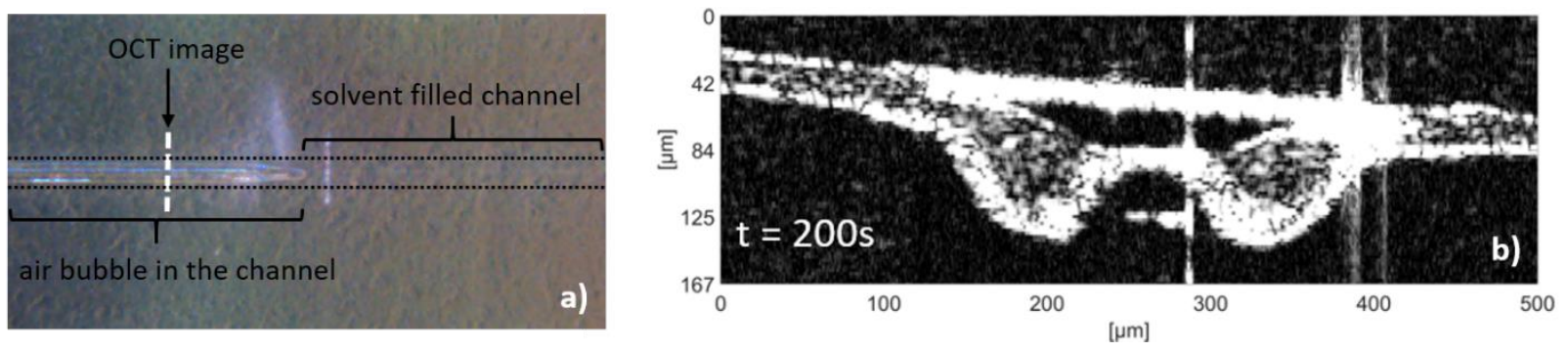

Figure 4 The dissolution process after $200 \mathrm{~s}$ at the point of the trapped air bubble in the microchannel. a) The location of the OCT measurement (white dashed line). For visibility, the microchannel is estimated by the black dashed lines. b) The corresponding cross-sectional OCT image shows the empty microchannel and the areas, where the solvent is diffused.

\section{Stress and strain simulation}

To evaluate the cause for the onset of the dissolution process from the bottom corners a simple model was applied to estimate stress and strain of the PMMA substrate due to hot-embossing process. The simulated stamp profile was obtained from the surface profile measurements (Veeco/Wyko NT3300) of the hotembossing stamp. The simulated channel depth was the same as in the samples. Due to the simplified conditions of hot-embossing simulation the absolute values are indicative. However, it is seen that the high 
von Mises stress concentration points appear at the corners (Fig. 5a). As can be expected, the largest plastic strains of the PMMA substrate are also located in the corners (Fig. 5b). Similar simulation of the stress and the strain distributions have been presented [12-14,34,35]. The simulation supports the conclusion that there is already a change in the morphology of PMMA substrate that originates from the roll-to-roll hot-embossing process and becomes visible during solvent lamination process.
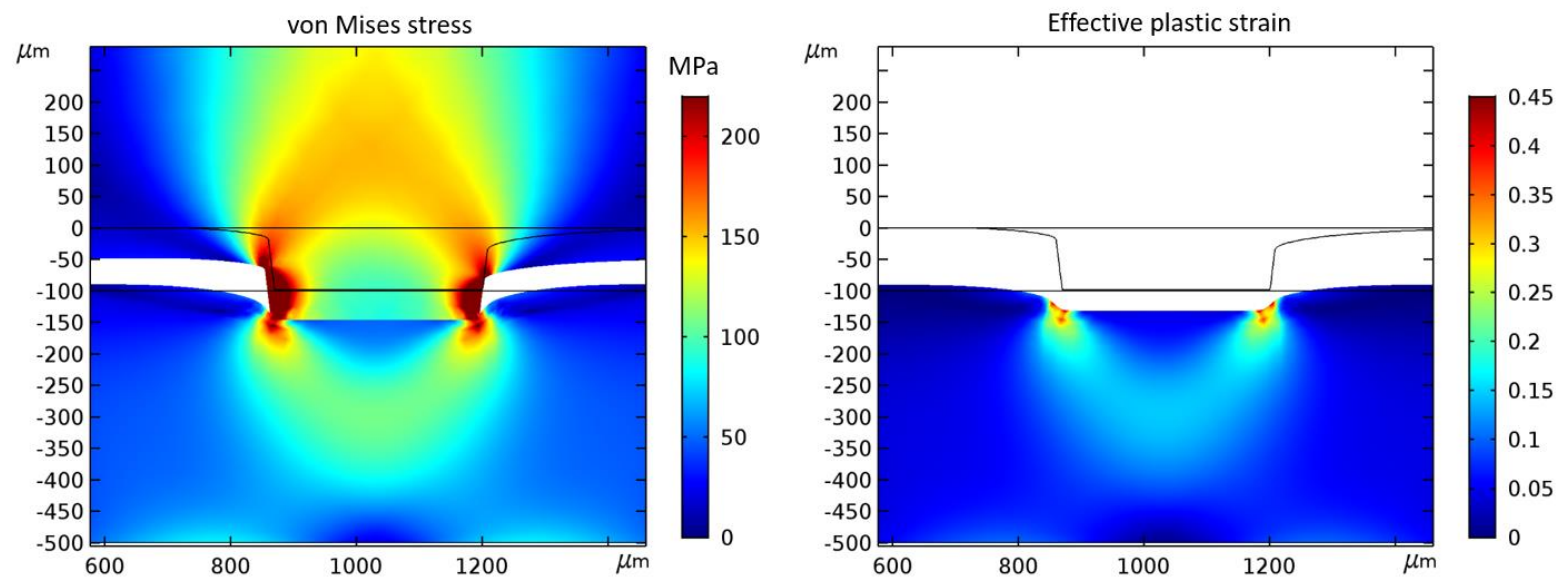

Figure 5 Simulations of von Mises stress distribution during hot embossing process and effective plastic strain after release of the mold.

\section{Performance studies}

The optical measurements shows that the channel walls contain porous structure that enabled leakage of fluids outside the channel into the substrate. This has negative impact on diagnostic test result e.g. in the case of immunoassay where detection antibodies labelled with dyes may leak into the cavities and cause unspesific signal enhancement.

In order to demonstrate the influence, fluorescently labelled antibody was added to the channel. The solution with goat anti-mouse IgG protein, labelled with AlexaFluor 488 fluorescence dye ( $41.4 \mu \mathrm{M}$ Invitrogen), was allowed to incubate within the channel for 5 minutes prior emptying the channel by suction. The channel was cut half through with a surgical knife and finally snapped. The cross-cut was imaged with a fluorescence microscope (Zeiss Axioskop Upright Fluorescence Microscope, Zeiss) using blue laser light for excitation. As seen in the fluorescence intensity image of the channel's cross-section (Fig. 6), the label has leaked to the substrate and resulted a strong fluorescence signal outside the channel. The fluorescence signal is localized in the areas were OCT observed the microvoids (Fig. 4). 


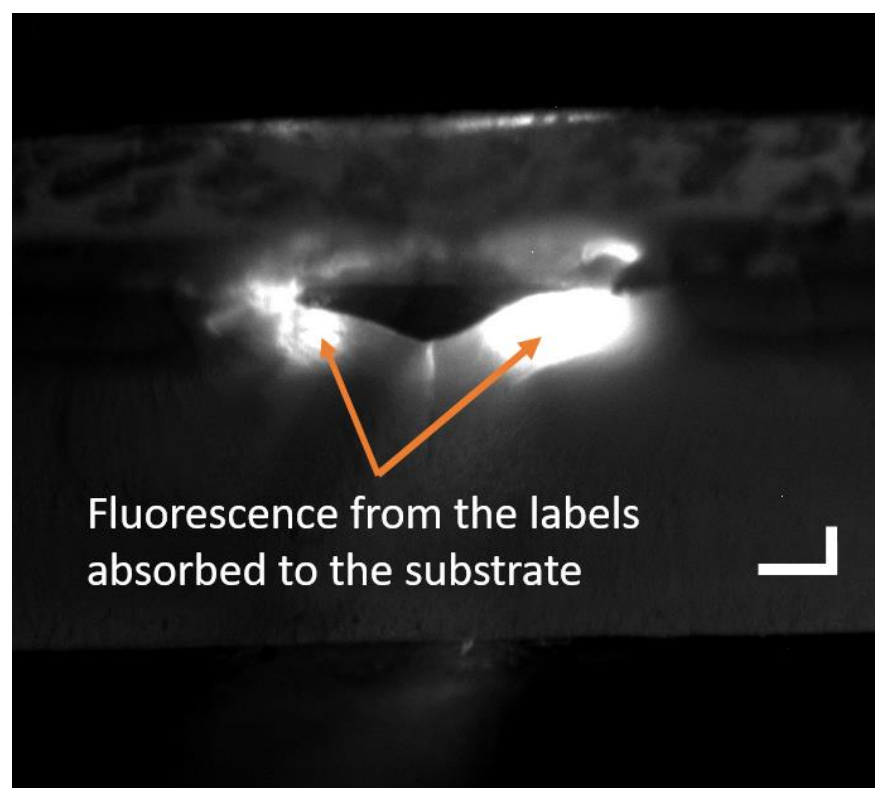

Figure 6 Fluorescence image of the empty channel's cross-cut after the channel was incubated for 5 minutes with the fluorescent solution. The high intensity locations at the bottom corners are result from the fluorescent markers being absorbed into the PMMA substrate. The scale bar is $50 \mu \mathrm{m}$.

\section{Conclusion}

In this work, OCT was successfully applied for fast and reliable characterization of solvent bonded microfluidics fabricated with roll-to-roll hot-embossing on PMMA. The OCT measurements showed that after the solvent lamination process the porous structures emerge beneath the microfluidic channel. The origin of the porous PMMA structures are assumed to be a result of the ceased swelling-evaporation processes. Furthermore, OCT was applied to measure solvent diffusion kinetics by tracking the sharp boundary of swollen layer and unpermeated glassy core. At the locations of the deepest porous structures the diffusion rate was the highest as well as, according to the simulations, the effective strain and von Mises stresses. The fluorescent labels were demonstrated to leak into the porous substrate, which will cause unspecific signal enhancement on the fluorescent signal detection and lead to a false reading. Characterization of a surface profile of a hotembossed microchannel is not enough to guarantee a quality of lidded microfluidics. The OCT is excellent tool to monitor quality of complete microfluidic devices and improve the repeatability of the microfluidic based bioassays. In addition, OCT can be applied to more fundamental studies of solvent diffusion kinetics in polymers.

\section{Acknowledgements}

European Regional Development Fund (project: Development of mass manufacturing methods for highly integrated disposable rapid diagnostic platforms, A70245) is gratefully acknowledged for supporting this work. In addition, the contribution of the last author of this paper is financially supported by Academy of Finland's 6Genesis Flagship (grant no. 318927).

\section{References}

1. Whitesides, G.M. The origins and the future of microfluidics. Nature 2006, 442, 368.

2. Becker, H.; Heim, U. Hot embossing as a method for the fabrication of polymer high aspect ratio structures. Sensors and Actuators A: Physical 2000, 83, 130-135, DOI //doi.org/10.1016/S09244247(00)00296-X. 
3. Wang, X.; Liedert, C.; Liedert, R.; Papautsky, I. A disposable, roll-to-roll hot-embossed inertial microfluidic device for size-based sorting of microbeads and cells. Lab Chip 2016, 16, 1821-1830, DOI 10.1039/C6LC00215C.

4. $\quad$ Liedert, R.; Amundsen, L.K.; Hokkanen, A.; Mäki, M.; Aittakorpi, A.; Pakanen, M.; Scherer, J.R.; Mathies, R.A.; Kurkinen, M.; Uusitalo, S.; Hakalahti, L.; Nevanen, T.K.; Siitari, H.; Söderlund, H. Disposable roll-to-roll hot embossed electrophoresis chip for detection of antibiotic resistance genemecA in bacteria. Lab Chip 2012, 12, 333-339, DOI 10.1039/C1LC20782B.

5. Wallow, T.I.; Morales, A.M.; Simmons, B.A.; Hunter, M.C.; Krafcik, K.L.; Domeier, L.A.; Sickafoose, S.M.; Patel, K.D.; Gardea, A. Low-distortion, high-strength bonding of thermoplastic microfluidic devices employing case-II diffusion-mediated permeant activation. Lab on a chip 2007, 7, 1825, DOI 10.1039/b710175a.

6. Tsao, C.; DeVoe, D. Bonding of thermoplastic polymer microfluidics. Microfluid Nanofluid 2009, 6, 116, DOI 10.1007/s10404-008-0361-x.

7. de Mello, A. Focus: Plastic fantastic? . Lab Chip 2002, 2, 36N, DOI 10.1039/B203828P.

8. Chen, P.; Duong, L.H. Novel solvent bonding method for thermoplastic microfluidic chips. Sensors \& Actuators: B. Chemical 2016, 237, 556-562, DOI 10.1016/j.snb.2016.06.135.

9. Masaro, L.; Zhu, X.X. Physical models of diffusion for polymer solutions, gels and solids. Progress in Polymer Science 1999, 24, 731-775, DOI 10.1016/S0079-6700(99)00016-7.

10. Thomas, N.; Windle, A.H. Transport of methanol in poly(methyl methacrylate). Polymer 1978, 19 , 255-265, DOI //doi.org/10.1016/0032-3861(78)90218-5.

11. Petropoulos, J.H.; Sanopoulou, M.; Papadokostaki, K.G. Physically insightful modeling of nonFickian kinetic regimes encountered in fundamental studies of isothermal sorption of swelling agents in polymeric media. European Polymer Journal 2011, 47, 2053-2062, DOI //doi.org/10.1016/j.eurpolymj.2011.07.019.

12. Liu, C.; Li, J.M.; Liu, J.S.; Wang, L.D. Deformation behavior of solid polymer during hot embossing process. Microelectronic Engineering 2010, 87, 200-207, DOI //doi.org/10.1016/j.mee.2009.07.014.

13. Narijauskaitè, B.; Palevičius, A.; Gaidys, R.; Janušas, G.; Šakalys, R. Polycarbonate as an ElastoPlastic Material Model for Simulation of the Microstructure Hot Imprint Process. Sensors 2013, 13, DOI 10.3390/s130911229.

14. Juang, Y.; Lee, L.J.; Koelling, K.W. Hot embossing in microfabrication. Part II: Rheological characterization and process analysis. Polym Eng Sci 2002, 42, 551-566, DOI 10.1002/pen.10971.

15. Peppas, N.A.; Wu, J.C.; von Meerwall, E.D. Mathematical Modeling and Experimental Characterization of Polymer Dissolution. Macromolecules 1994, 27, 5626-5638, DOI 10.1021/ma00098a017.

16. Herman, M.F.; Edwards, S.F. A reptation model for polymer dissolution. Macromolecules 1990, 23, 3662-3671, DOI 10.1021/ma00217a020.

17. Miller-Chou, B.A.; Koenig, J.L. A review of polymer dissolution. Progress in Polymer Science 2003, 28, 1223-1270, DOI //doi.org/10.1016/S0079-6700(03)00045-5. 
18. Stamatialis, D.F.; Sanopoulou, M.; Raptis, I. Swelling and dissolution behavior of poly(methyl methacrylate) films in methyl ethyl ketone/methyl alcohol mixtures studied by optical techniques. $J$ Appl Polym Sci 2002, 83, 2823-2834, DOI 10.1002/app.10258.

19. Lauri, J.; Czajkowski, J.; Myllylä, R.; Fabritius, T. Measuring flow dynamics in a microfluidic chip using optical coherence tomography with $1 \mu \mathrm{m}$ axial resolution. Flow Meas Instrum 2015, 43, 1-5, DOI //dx.doi.org/10.1016/j.flowmeasinst.2015.02.001.

20. Ahn, Y.; Jung, W.; Chen, Z. Optical sectioning for microfluidics: secondary flow and mixing in a meandering microchannel. Lab Chip 2008, 8, 125-133, DOI 10.1039/B713626A.

21. Bukowska, D.M.; Derzsi, L.; Tamborski, S.; Szkulmowski, M.; Garstecki, P.; Wojtkowski, M. Assessment of the flow velocity of blood cells in a microfluidic device using joint spectral and time domain optical coherence tomography. Opt Express 2013, 21, 24025-24038, DOI //dx.doi.org/10.1364/OE.21.024025.

22. Wang, L.; Xu, W.; Bachman, M.; Li, G.P.; Chen, Z. Phase-resolved optical Doppler tomography for imaging flow dynamics in microfluidic channels. Appl Phys Lett 2004, 85, 1855-1857, DOI //dx.doi.org/10.1063/1.1785854.

23. Fabritius, T.; Myllylä, R.; Makita, S.; Yasuno, Y. Wettability characterization method based on optical coherence tomography imaging. Opt Express 2010, 18, 22859-22866, DOI 10.1364/OE.18.022859.

24. Koponen, A.; Lauri, J.; Haavisto, S.; Fabritius, T. Rheological and Flocculation Analysis of Microfibrillated Cellulose Suspension Using Optical Coherence Tomography. Applied Sciences 2018, 8, DOI 10.3390/app8050755.

25. Lauri, J.; Koponen, A.; Haavisto, S.; Czajkowski, J.; Fabritius, T. Analysis of rheology and wall depletion of microfibrillated cellulose suspension using optical coherence tomography. Cellulose 2017, 24, 4715-4728, DOI 10.1007/s10570-017-1493-5.

26. Lauri, J.; Bykov, A.; Fabritius, T. Quantification of cell-free layer thickness and cell distribution of blood by optical coherence tomography. 2016, 21,4 .

27. Zysk, A.M.; Nguyen, F.T.; Oldenburg, A.L.; Marks, D.L.; Boppart, S.A. Optical coherence tomography: a review of clinical development from bench to bedside. 2007, 12, 21.

28. Golde, J.; Kirsten, L.; Schnabel, C.; Walther, J.; Koch, E. Optical Coherence Tomography for NDE. In Handbook of Advanced Non-Destructive Evaluation; Ida, N.; Meyendorf, N., Eds.; Springer International Publishing: Cham, 2018; pp. 1-44.

29. Czajkowski, J.; Fabritius, T.; Ulański, J.; Marszałek, T.; Gazicki-Lipman, M.; Nosal, A.; Śliż, R.; Alarousu, E.; Prykäri, T.; Myllylä, R.; Jabbour, G. Ultra-high resolution optical coherence tomography for encapsulation quality inspection. Applied Physics B 2011, 105, 649-657, DOI 10.1007/s00340-011$4699-5$.

30. Hierzenberger, P.; Leiss-Holzinger, E.; Heise, B.; Stifter, D.; Eder, G. In-Situ Optical Coherence Tomography (OCT) for the Time-Resolved Investigation of Crystallization Processes in Polymers. Macromolecules 2014, 47, 2072-2079, DOI 10.1021/ma4023839.

31. Zaleski, R.; Krasucka, P.; Skrzypiec, K.; Goworek, J. Macro- and Nanoscopic Studies of Porous Polymer Swelling. Macromolecules 2017, 50, 5080-5089, DOI 10.1021/acs.macromol.7b00820. 
32. Jeong, U.; Im, S.H.; Camargo, P.H.C.; Kim, J.H.; Xia, Y. Microscale Fish Bowls: A New Class of Latex Particles with Hollow Interiors and Engineered Porous Structures in Their Surfaces. Langmuir 2007, 23, 10968-10975, DOI 10.1021/la702324q.

33. Han, J.; Song, G.; Guo, R. Fabrication of polymer hollow nanospheres by a swelling-evaporation strategy. J Polym Sci A Polym Chem 2007, 45, 2638-2645, DOI 10.1002/pola.22023.

34. Park, J.M.; Kang, T.G.; Park, S.J. Numerical simulation of hot embossing filling stage using a viscoelastic constitutive model. Korea-Australia Rheology Journal 2011, 23, 139, DOI 10.1007/s13367-011-0017-3.

35. Peng, L.; Deng, Y.; Yi, P.; Lai, X. Micro hot embossing of thermoplastic polymers: a review. $J$ Micromech Microengineering 2014, 24, 013001. 